\title{
Innovative Low-Cost Plastic Optical Fiber Sensors for Gas Monitoring
}

\author{
Maen Ishtaiwi ${ }^{*}, 1$, Marco Parvis ${ }^{2}$, Sabrina Grassini ${ }^{3}$ and Alberto Vallan 4 \\ ${ }^{*}, 1$ Politecnico di Torino, Department of Electronics and Telecommunications, Corso Duca degli Abruzzi 24, \\ Torino; E-Mail: maen.ishtaiwi@ polito.it ; Tel.: +39 011090 4114; Fax: +39 0110904099. \\ ${ }^{2,4}$ Politecnico di Torino, Department of Electronics and Telecommunications, Corso Duca degli Abruzzi 24, \\ Torino; E-Mails: marco.parvis@ polito.it ; alberto.vallan@ polito.it \\ ${ }^{3}$ Politecnico di Torino, Department of Applied Science and Technology, Corso Duca degli Abruzzi 24, Torino; \\ E-Mail: $\underline{\text { sabrina.grassini@polito.it }}$
}

Published: 1 June 2014

\begin{abstract}
In this study, a new simple and low-cost plastic optical fiber sensor for the measurements of low concentration HF vapors is described. The sensitivity to the HF vapors obtained by depositing a thin glass-like layer onto the PMMA core fiber after removing the fluorinated polymer cladding via dipping the fiber into ethyl acetate for a short period, not more than 40 second. Moreover, in order to enhance the fiber sensitivity, low pressure plasma treatments have been carried out in $\mathrm{O}_{2}$ and $\mathrm{Ar}$ gas mixtures on polymeric optical core fibers in order to increase the core surface area without damaging the PMMA core fiber. The reaction of glass-like layer with the HF vapor alters the fiber light transmission and is detected by means of a wide-band photodiode. POF sensors can find relevant applications for monitoring people exposed to unhealthy atmospheres in civil and industrial fields, and for monitoring the pollutants in gaseous mixtures. Several prototypes of fiber sensors have been fabricated and characterized in the reaction chamber. The obtained results demonstrating the capability to detect low concentration of HF vapors with high sensitivity.
\end{abstract}

Keywords: POF sensor; HF detector; Gas monitoring.

\section{Introduction}

Gas monitoring is an area of increasing interest, especially to control volatiles that can be harmful to human health. Pollution caused by chemicals such as organic gases is well-known problem and its 
monitoring is a challenge which forces the development of new sensitive and specific sensors. Moreover, in some specific application such as Resistive Plate Counter (RPC) [1] which are gas detectors extensively used in nuclear and particle physics for the detection and timing of muon particles, the absence of contaminants is important in all gas detectors but is critical in the case of RPCs since their presence is responsible for an increase of the dark current until the RPCs become not usable.

Usually electrochemical gas sensors are used to detect toxic gases, but these types of sensors are sensitive to the instantaneous gas concentration and thus require integrating the sensor readings to compute the cumulative exposure; a method that may lead to important errors. On the other hand, several types of sensors are commercially available to detect the pollutants (e.g. galvanic-type sensors that are based on $\mathrm{MnO}_{2}$ gas-diffusion electrodes [2]), which are used for hydrohalogen $(\mathrm{HBr}, \mathrm{HCl}$, and $\mathrm{HF}$ ) concentration measurement in the air. Other examples are tin-dioxide-based gas sensors, which can detect hydrogen fluoride traces at part per billion ( $\mathrm{ppb}$ ) concentration [3, 4]. Most of these sensors, however, have a high cost and not able to work in the presence of high intensity electric filed.

In this research, low cost intrinsic sensors based on Plastic Optical Fibers (POFs) made of polymethyl-methacrylate (PMMA) [5, 6] could be an interesting alternative. Plastic optical fibers can be used to develop intrinsic sensors owing to several different principles such as light attenuation change due to pollutant absorption into the core, and light losses due to fiber bending [7, 8]. From this point of view, POFs have a remarkable evanescent field, which extend outside the core and can be used to develop intrinsic sensors based on the changes in the propagation losses connected to the interaction of the evanescent field with the surrounding media. The sensor has several advantages, mainly the ability to evaluate directly the cumulative exposure and the transducer is based on a plastic optical fiber and exploits a non-reversible chemical reaction.

\section{Experimental Section}

\subsection{POF sensors preparation}

The plastic optical fiber used in this study is a standard, commercial step-index, highly multimode and low cost. The fiber has a cladding-core structure made of a fluoro-polymer and super pure polymethylmethacrylate (PMMA), respectively. The fiber has a cladding diameter of about $1000 \mu \mathrm{m}$ while core diameter is about $980 \mu \mathrm{m}$. The cladding-core structure is protected in a jacket layer. The removal of the cladding is an essential step in POF sensors development in order to take advantage of evanescent field and the PMMA core exposed. Low pressure plasma etching can directly modify the PMMA core surface then the sensitive thin layer (glass-like) can interact with evanescent field. The fluoro-polymer can be removed by chemical process by dipping the fiber in ethyl-acetate solution for short period, not more than about 40 second in order to avoid damaging the PMMA core, the cladding is nearly completely dissolved into the solvent and the fluoro-polymer remained onto the fiber which can be manually removed with soft paper.

The sensor prototype structure is composed of plastic fiber with length about of $10 \mathrm{~cm}$, a light emitting diode (LED) as light source at the one end, and a photodiode (PD) that able to collect the light at the fiber end. The PD and LED cupolas centers are drilled with $1 \mathrm{~mm}$ holes. Fiber ends were 
polished with grinding paper, then a liquid PMMA glue is used to bond the bare fiber which is inserted into the holes. The fiber is then cured in an oven at $60-70^{\circ} \mathrm{C}$ for 60 hours to ensure complete polymerization of the liquid PMMA. The complete fiber assembly is set to PMMA support so that the fiber is in a fixed position. The sensor is sensitive to environmental conditions specially the changes in temperature, so several types of support has been designed such as Polyvinyl Chloride (PVC) support, standard perfboard support, and polymethylmethacrylate (PMMA) support. The best result has been obtained by using the PMMA support, since the plastic fiber is made of PMMA whereas perfboard is made of henolic resin and metal and PVC is made of vinyl chloride monomer, therefore they have different thermal expansion coefficients and some bending is due to temperature variation. The complete sensors assembly is then insert in plasma reactor to modify the fiber surface (PMMA core) and then to deposit thin film onto the core. Moreover, Teflon insulated wires are used to connect the LED and PD. The connections, LED and PD were painted with a black HF resistant paint to avoid the troubles due to the effect of environment light which could travel from LED to the PD outside the fiber.

\subsection{Coating plastic optical fiber}

The thin layer that has to be coated onto the fiber core must be capable to react with the sensed quantity (HF vapors) and the reaction able to alter the capability of the fiber of transmitting the light. On the other hand, choosing the sensitive layer must be taken in the account the probability reacting to other quantities present in the surrounding environment, which could effect on the transmission light. In the case of fluoride ions detection, glass has been selected to deposit on the fiber core due to the capability of fluoride ions to attack the glass layer. $\mathrm{SiO}_{x}$ has a higher refractive index than the PMMA core and it is a good advantage for affecting on light transmission. In facts, it is known that HF reacts with glass leading to the formation of silicon tetrafluoride $\left(\mathrm{SiF}_{4}\right)$ :

$$
\mathrm{SiO}_{2}+4 \mathrm{HF} \rightarrow \mathrm{SiF}_{4}+2 \mathrm{H}_{2} \mathrm{O}
$$

$\mathrm{SiO}_{\mathrm{x}}$ thin film were deposited by using Plasma Enhanced Chemical Vapor Deposition (PECVD) of organosilicon monomers onto a fiber by using a plasma fed with Tetraethyl orthosilicate (TEOS), $\mathrm{O}_{2}$ and Ar. In order to get the glass layer cable to react with HF vapors two parameters must be considered: the deposition time and the gas composition. The deposition time of 20 min to obtain a thickness of about $200 \mathrm{~nm}$. A gas mixture ratio (Ar:TEOS: $\mathrm{O}_{2}=20: 1: 20$ ) at the pressure $5 \mathrm{~Pa}$ and with an input power of $50 \mathrm{~W}$ was used for the deposition to obtain a mostly inorganic $\mathrm{SiO}_{\mathrm{x}}$ film.

\section{Results and Discussion}

A test has been performed to investigate the effect of $\mathrm{HF}$ vapors on the $\mathrm{SiO}_{\mathrm{x}}$ coated $\mathrm{POF}$ sensor by employing the measuring setup sketched in Figure 1. The sensor assembly and A Pt100 which used to monitor the temperature are inserted inside the reaction chamber. HF vapor generation is obtained by using a small PFTE bowl containing a few milliliters of HF solutions. The partial pressure of HF vapors can be computed using the Antoine equation [9] and the coefficients can be found either on the NIST web site [10] or computed from partial pressure experimental measurements [11].

\subsection{Coted fiber response to HF}


The sensor system measures the hydrogen fluoride exposition $E_{H F}$ from end of deposition time $t_{0}$ to measure instant $t$. Fiber light transmittance $T_{\text {fiber }}$ is computed as ratio between light source and destination currents $I_{i n}$ and $I_{\text {out }}$, keeping into account the system offset currents $I_{\text {in }, 0}$ and $I_{\text {out }, 0}$. Eventually $T_{\text {fiber }}$ is used to estimate $E_{H F}$. Instantaneous HF concentration $e_{H F(t)}$ is not directly obtained:

$$
E_{H F(t)}=\int_{t_{0}}^{t} e_{H F}\left(t^{\prime}\right) d t^{\prime}, E_{H F}(t) \propto T_{\text {fiber }}(t)=\frac{I_{\text {out }}(t)-I_{\text {out }, 0}}{I_{\text {in }}(t)-I_{\text {in }, 0}}
$$

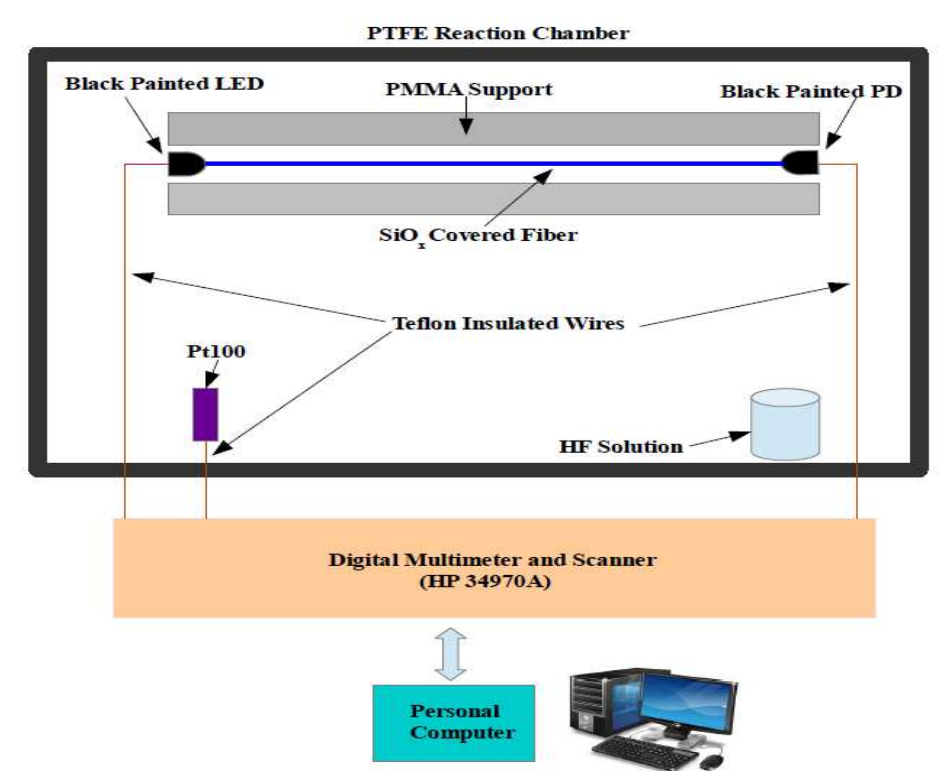

Figure 1. Measurement setup: The sensor assembly is composed of a coated fiber, a black-painted LED, a black painted photodiode, and a PMMA support. A Pt100 is used for temperature measurements. A PC is connected to a DMMA scanner via IEEE488 interface. The reaction chamber is made of polytetrafluoroethylene (PTFE) which is HF-resistant. The HF vapors are generated by inserting a small PTFE bowl containing a calibrated solution of HF into the reaction chamber.

Figure 2 shows the result of an exposition of about 27 hours to the HF vapors produced. The hydrogen fluoride vapors are introduced at the beginning and then let in place for the remaining time. By exposing the fiber to this concentration, the photodiode current increases as the time passes until a plateau. After exposure of about 30000 ppm per hour, the current reaches the saturation which appears when the current reaches about $117 \mathrm{nA}$. The temperature during the test is not controlled and changes in the range of $19^{\circ} \mathrm{C}$ to $22.5^{\circ} \mathrm{C}$ with a minimal effect on the result.

Figure 3 shows, as an example, the result of an exposure test. The figure shows the temperature measured by the Pt100 sensor and changes in the range of $17^{\circ} \mathrm{C}$ to $21.5^{\circ} \mathrm{C}$. The middle traces show the exposure to HF vapors computed as the integral of the vapor concentration. The bottom trace shows the fiber transmittance ratio normalized to its initial value. The result obtained during this test is shown that the fiber has an output that follows the exposure until saturation, which appears when the transmission ratio increases about 5 times. After about 90 hours, the transmission ratio reaches a plateau.

\subsection{Fiber nano-texturing effect}



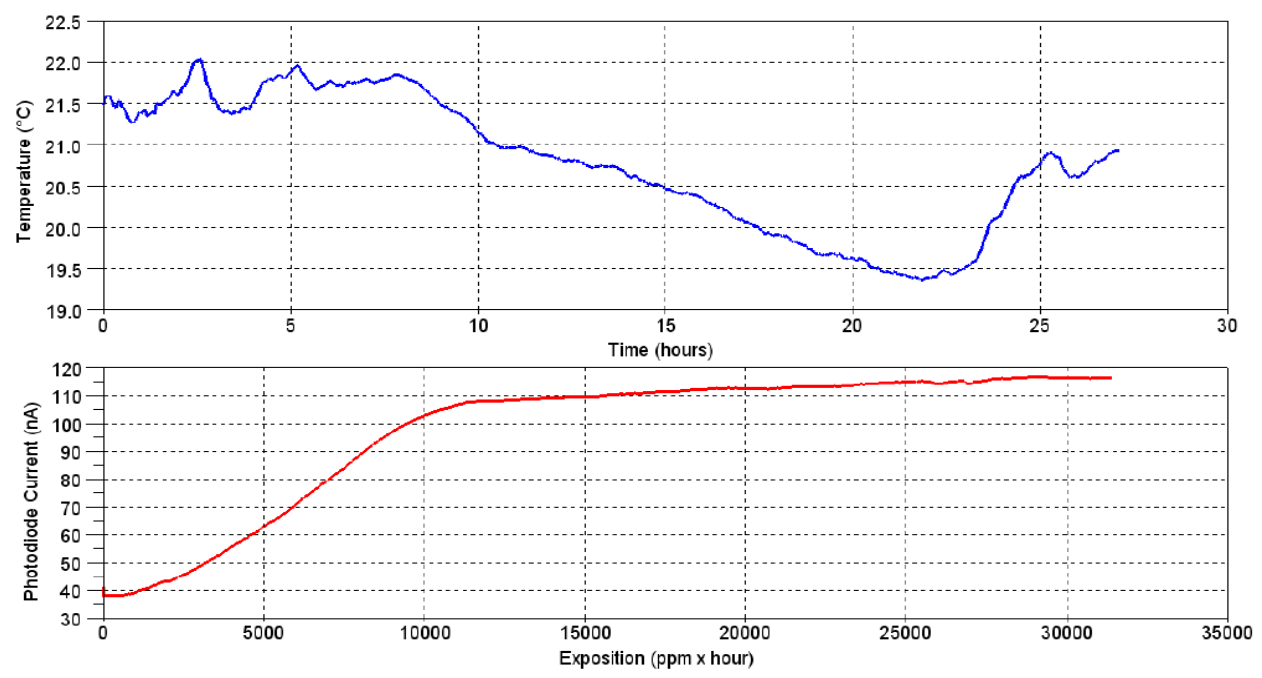

Figure 2. Photodiode current change due to the $\mathrm{HF}$ reaction on the $\mathrm{SiO}_{\mathrm{x}}$ layer deposited on the fiber. It is clear how the degradation of $\mathrm{SiO}_{\mathrm{x}}$ layer due to $\mathrm{HF}$ results in an increase of the photodiode current. The current increases until the external coating reaches a stable degradation state after about $25 \mathrm{~h}$.
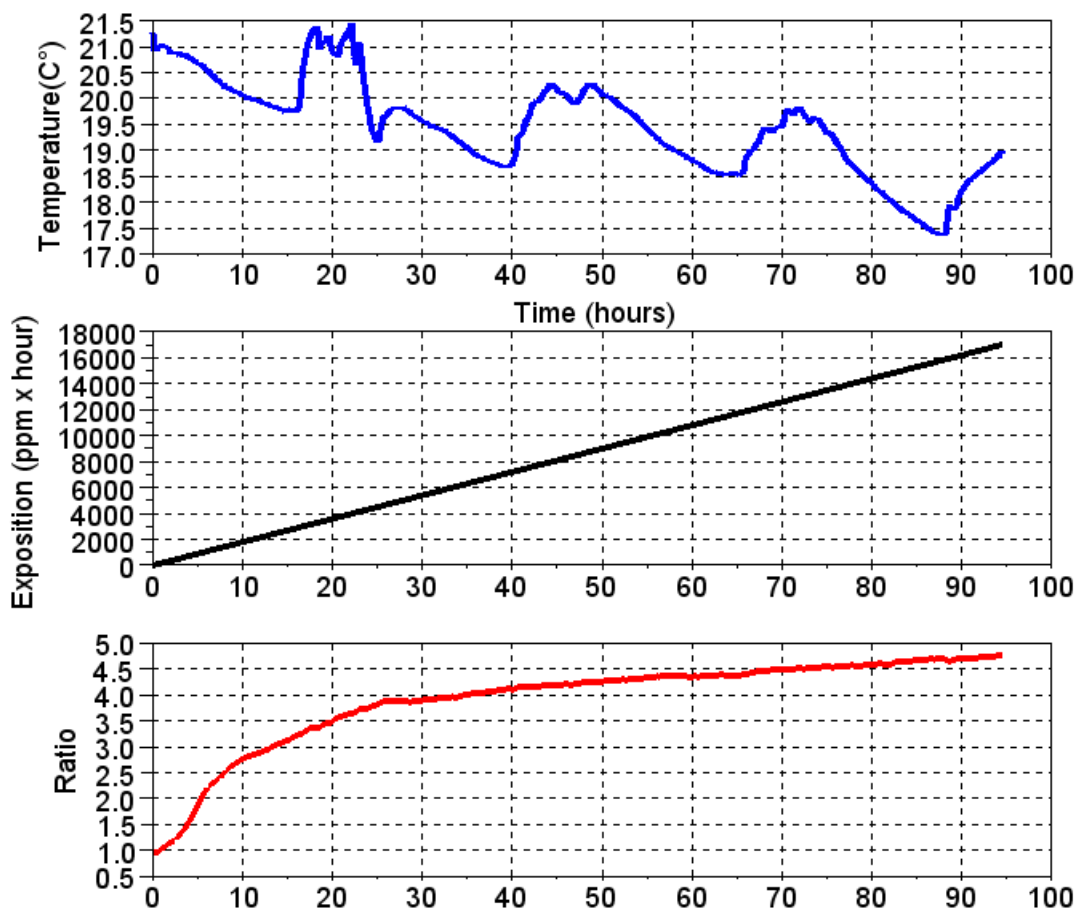

Figure 3. Coated fiber response to HF exposition. Top trace: The temperature during the test measured with the Pt100 sensor. Middle trace: exposition computed as the integral of the vapor concentration. Bottom trace: fiber transmittance ratio normalized to its initial value.

The plasma nano-texturing has been exploited to enhance the sensitivity of plastic optical fibers sensors. The plasma treatments have been performed in $\mathrm{O}_{2}$ and $\mathrm{Ar}$ gas mixtures, in different experimental conditions, on polymeric optical fibers in order to increase the surface area without damaging the fiber structure. The effect of the nano-texturing treatment is mainly the creation of nanostructures whose dimensions and distance increase light scattering and thus leads to a 
transmittance ratio decrease on the order of 5\%. The nano-textured PMMA core fiber, which has a high equivalent surface area with respect to the non treated fiber, significantly increases the sensors sensitivity compared to untreated PMMA surface core. This is clear shown in Table 1 where the nanotextured sensor has a higher transmission rate change.

\begin{tabular}{|c|c|c|c|c|}
\hline & Uncladding & Nano-textured PMMA & Coating glass-like & Exposing to HF \\
\hline Nano-textured fiber & 1 & 0.96 & 0.069 & 0.69 \\
\hline Non treated fiber & 1 & & 0.059 & 0.11 \\
\hline
\end{tabular}

Table 1. Normalized transmittance ratio for the two different fiber sensors.

\section{Conclusions}

In this paper an innovative sensor based on a low cost plastic optical fiber and suitable for HF vapor monitoring has been described. Preliminary characterizations have demonstrated a good repeatability and a fast response. Therefore the developed sensor can be successfully proposed to monitor the total exposure over time to fluoride vapors. The experiments described in this paper, have shown that the light transmitted by the fiber changes as soon as the $\mathrm{SiO}_{\mathrm{x}}$ is attacked by $\mathrm{HF}$ vapors; and this change is higher in case of the nano-textured PMMA core fiber. The authors are now working on the characterization procedure of sensitivity enhancement, i.e., the optimization low pressure plasma treatments parameters such as the gas mixtures and treatment time.

\section{References}

1. R. Santonico and R. Cardarelli, Development of resistive plate counters, Nucl. Instrum. Meth. 187 (1981) 377.

2. V.P. Chviruk, O.V. Linuycheva and E.M. Zaverach. Galvanic-type electrochemical sensor for determining the content of hydrohalogens in the air, Sensors Actuator. B 92 (2003) 60

3. F. Bergera, J.B. Sancheza and O. Heintzb, Detection of hydrogen fluoride using $\mathrm{SnO}_{2}$-based gas sensors: understanding of the reactional mechanism, Sensors Actuator. B 143 (2009) 152

4. Y. Suna, L. Chena, F. Zhangb, D. Li, H. Pana and J. Yec, First-principles studies of HF molecule adsorption on intrinsic graphene and Al-doped graphene, Solid State Commun. 150 (2010) 1906

5. J.A. Buck, Fundamentals of optical fibers, $2^{\text {nd }}$ edition, Wiley and Sons, Inc., New York U.S.A. (2004).

6. J. Zubia and J. Arrue, Plastic optical fibers: an introduction to their technological processes and application, Opt. Fiber Technol. 7 (2001) 101.

7. B. Ruchti et al., Development of new scintillating fiber detectors for high energy physics applications, IEEE Trans. Nucl. Sci. 36 (1989) 146.

8. G. Perrone and A. Vallan, A low-cost optical sensor for noncontact vibration measurements, IEEE Trans. Instrum. Meas. 58 (2009) 1650.

9. C. Antoine, Tensions des vapeurs; nouvelle relation entre les tensions et les temp`e, Compt. Rend. Acad. Sci. 107 (1888).

10. http://webbook.nist.gov/chemistry/ , (Sept. 2011).

11. Hydrofluoric acid properties, available at http://honeywell.com, (Sept. 2011).

(C) 2014 by the authors; licensee MDPI, Basel, Switzerland. This article is an open access article distributed under the terms and conditions of the Creative Commons Attribution license (http://creativecommons.org/licenses/by/3.0/). 